\title{
Noradrenaline and Dopamine Efflux in the Prefrontal Cortex in Relation to Appetitive Classical Conditioning
}

\author{
Susana Mingote, Jan P. C. de Bruin, and Matthijs G. P. Feenstra \\ Netherlands Institute for Brain Research, 1105 AZ, Amsterdam, The Netherlands
}

We trained rats to learn that an auditory stimulus predicted delivery of reward pellets in the Skinner box. After $2 \mathrm{~d}$ of training, we measured changes in efflux of noradrenaline (NA) and dopamine (DA) in the medial prefrontal cortex using microdialysis on the third day. Animals were subjected to a normal rewarded session and an extinction session, in which the auditory stimulus was presented alone. In the rewarded session, both NA and DA efflux were increased, but in extinction, only NA was activated. The data suggest that NA has a role in the reaction to reward-predicting stimuli, which complements that of DA.

Key words: prefrontal; conditioning; reward; dopamine; noradrenaline; motivation; dialysis

\section{Introduction}

The mesocorticolimbic dopamine (DA) system plays a critical role in motivational processes (Robbins and Everitt, 1996; Kalivas and Nakamura, 1999). DA also exerts an important modulatory influence on cognitive functions typically attributed to the prefrontal cortex (PFC), such as working memory (Arnsten, 1997). The tonic release provides an activation of DA receptors in the PFC that may be related to working memory in an inverted U-shaped manner (Goldman-Rakic et al., 2000). In addition, phasic activation may favor the processing of stimuli that are of direct importance to the animal, given its present needs and preferences.

Evidence from in vivo microdialysis studies indicates that appetitive emotional stimuli can induce such a phasic activation of prefrontal DA release (for review, see Feenstra, 2000). This applies not only to direct, unconditioned stimuli (USs) but also to conditioned stimuli (CSs) that predict reward presentation through classical (pavlovian) conditioning. Consistent with the hypothesis that DA plays a role in appetitively motivated behavior, Bassareo and Di Chiara (1997) and Ahn and Phillips (1999) reported conditioned DA efflux in the PFC.

Although noradrenaline (NA), like DA, may have similar importance as a modulator of prefrontal functions (Arnsten, 1997), the role of noradrenergic innervation of the PFC in the processing of reward-related information has essentially been neglected. We found one direct indication of its activation by stimuli related to natural rewards (Sara and Segal, 1991), while only recently, Drouin et al. (2002) and Ventura et al. (2003) uncovered a role for NA in the motivational effects of addictive drugs. Addressing

\footnotetext{
Received 0ct. 7, 2003; revised Jan. 14, 2004; accepted Jan. 14, 2004.

This research was supported by the Leonardo da Vinci European Community Program.

Correspondence should be addressed to Matthijs Feenstra, Netherlands Institute for Brain Research, Meibergreef

33, 1105 Amsterdam Z0, The Netherlands. E-mail: M.Feenstra@nih.knaw.nl.

S. Mingote's present address: Division of Behavioral Neuroscience, Department of Psychology, University of Connecticut, Storrs, CT 06269.

DOI:10.1523/JNEUROSCI.4547-03.2004

Copyright $\odot 2004$ Society for Neuroscience $\quad$ 0270-6474/04/242475-06\$15.00/0
}

this subject, we designed experiments to directly compare the activation of prefrontal NA and DA efflux by classical conditioning. Rats were trained in a fully controlled environment (Skinner box) to associate a neutral auditory stimulus with the receipt of an unconditioned stimulus. Applying this approach, we reported that presentation of aversive conditioned stimuli increased both prefrontal NA and DA in vivo efflux (Feenstra et al., 2001), indicating a role for both catecholamines in coding the significance value of stimuli. Therefore, we tested the hypothesis that presentation of appetitive conditioned stimuli would also lead to activation of both catecholamine afferents to the PFC.

\section{Materials and Methods}

All experiments were approved by the Animal Experimentation Committee of the Royal Netherlands Academy of Arts and Sciences and were conducted in agreement with Dutch laws (Wet op de Dierproeven, 1996) and European regulations (Guideline 86/609/EEC).

Subjects. Male Wistar rats (Harlan CPB, Zeist, The Netherlands) weighing 100-125 gm at arrival were socially housed in groups of four under a reversed light/dark cycle (dimmed red light from 7:00 A.M. to 7:00 P.M.). They were kept for at least 3 weeks with food and water available ad libitum and were handled frequently. This schedule was maintained for the animals in experiment 1 up to the day of surgery. For the animals of experiment 2, food was taken away in the afternoon $5 \mathrm{~d}$ before surgery and was subsequently restricted to $16 \mathrm{gm}$ per rat per day. The weights of these rats were reduced to $\sim 90 \%$ of free-feeding weights.

Rats were anesthetized with chloralhydrate (3.5\% solution in saline; $400 \mathrm{mg} / \mathrm{kg}$, i.p.) with additional local anesthesia via lidocaine (6\% solution in saline). Under aseptic conditions, two microdialysis probes with 4 mm exposed length of Hospal AN69 dialysis membrane were placed bilaterally in the medial PFC at an angle of $12^{\circ}$ (coordinates in millimeters from bregma: anterior, +3.0 ; lateral, \pm 1.8 ; ventral, -5.5$)$, as described previously (Feenstra et al., 1998).

After recovery from anesthesia, each rat was placed in its individual Perspex cage $(25 \times 25 \times 32 \mathrm{~cm})$ with ad libitum access to food and water. On the following day, food was again restricted in experiment 2, and behavioral tests were started. Weights at the time of testing were between 250 and $350 \mathrm{gm}$.

Behavioral apparatus. Training and testing were conducted in dialysiscompatible Skinner boxes measuring $29.2 \times 24.1 \times 21 \mathrm{~cm}$ (MED Asso- 
ciates, St. Albans, VT), mounted within sound- and light-attenuating chambers, and dimly illuminated by a light oriented toward the ceiling. All Skinner boxes were equipped with a food dispenser from which pellets (Noyes formula P; 45 mg; Sandown Scientific, Hampton, UK) could be delivered and a wall-mounted loudspeaker through which an auditory stimulus (white noise, $25-30 \mathrm{~dB}$ measured at $10 \mathrm{~cm}$ distance) could be presented. Because of the extended stay in the boxes, water bottles were present. Behavioral responses before and during the CS presentation were recorded by the following: (1) an infrared photo beam that could detect each rat's head entry into the food dispenser (nose poke) and (2) an infrared movement detector mounted on the ceiling that recorded the general locomotor activity throughout the box. The recorded measures included the number of nose pokes, latency until the first nose poke after the start of CS presentation, and general locomotor activity.

Neurochemical apparatus. On the third day after surgery (microdialysis day), implanted probes were connected in series (Feenstra et al., 2002) using flexible polyetheretherketone (inner diameter, $0.13 \mathrm{~mm}$ ) tubing to a Tsumura (TCS 2-23) quartz-lined dual-channel swivel (Pronexus, Skärholmen, Sweden), and Ringer's solution (in mm: $145 \mathrm{NaCl}, 2.7 \mathrm{KCl}$, $1.2 \mathrm{CaCl}_{2}, 1.0 \mathrm{MgCl}_{2}$ ) was perfused at a constant rate of $3 \mu \mathrm{l} / \mathrm{min}$. Dialysis samples were automatically injected every $16 \mathrm{~min}$ into the HPLC system, as described by Feenstra et al. (1998).

Experimental procedures. On the 2 consecutive days after surgery, the rats were trained in Skinner boxes. On the third day, training was combined with on-line microdialysis measurements. Three different groups of rats were tested: paired, unpaired, and controls.

The paired group was trained to associate a neutral stimulus (white noise, CS) with pellet delivery (appetitive US). On the first day, rats were placed in the Skinner boxes with five pellets available in the food dispenser. They were allowed to explore the box for $90 \mathrm{~min}$. After this adaptation period, three normal acquisition sessions (CS plus US) were given. Each session consisted of six presentations of a $10 \mathrm{sec}$ noise, separated by random intervals ranging between 100 and $200 \mathrm{sec}$. In the final second of the noise, two (experiment 1) or four (experiment 2) pellets were delivered. Session duration was $16 \mathrm{~min}$, and intersession intervals were variable, averaging $60 \mathrm{~min}$. The animals were returned to their home cages $1 \mathrm{hr}$ after the last session. On the following day, the same training schedule was used but without the adaptation period. On the third day, NA and DA efflux were measured on-line throughout the day. A CS plus US session was given when the efflux had stabilized. Two hours later, a CS-alone (extinction) session was given, in which the animals were subjected to six unrewarded CS presentations. Behavioral scores are now indicative of the strength of the associative learning process.

The unpaired group was subjected to the same schedule of sessions during the three testing days, with one exception: in each session, the CS was presented explicitly unpaired with the US (stimuli separated by a random interval of 50-100 sec). The rats of the control group had similar sessions on all $3 \mathrm{~d}$, consisting only of CS presentations.

Histology. Coronal $(20 \mu \mathrm{m})$ cryostat sections were stained with thionine and examined for the precise location of the microdialysis probes. Data from rats with incorrectly placed probes were not reported.

Data analysis. For nose poke and locomotor activity data, differences were calculated between the values measured during the $10 \mathrm{sec}$ of CS presentation and the $10 \mathrm{sec}$ immediately before that. Latencies were transformed into ${ }^{10} \log$ values.

Statistical analyses of these data were first conducted for the full experiment (days 1-3) and later separately for day 3. ANOVA with repeated measures [using the Huynh-Feldt $\epsilon$ for correction (Norušis, 1990)] had session as the within-subject factor and group as the between-subject factor. When indicated, between-subject effects were analyzed using Student-Newman-Keuls (SNK) tests. ANOVA with repeated measures for separate groups was followed, when indicated, by simple contrast analysis to compare the data of the first training session with those of successive sessions. The level of significance was $p<0.05$ in all circumstances.

Changes in neurochemical concentrations were expressed as percentages, with the mean of the first three samples preceding the stimulus presentation set at $100 \%$ as basal level.

The statistical analyses of the microdialysis data were conducted separately for the final CS plus US session and for the CS-alone session.
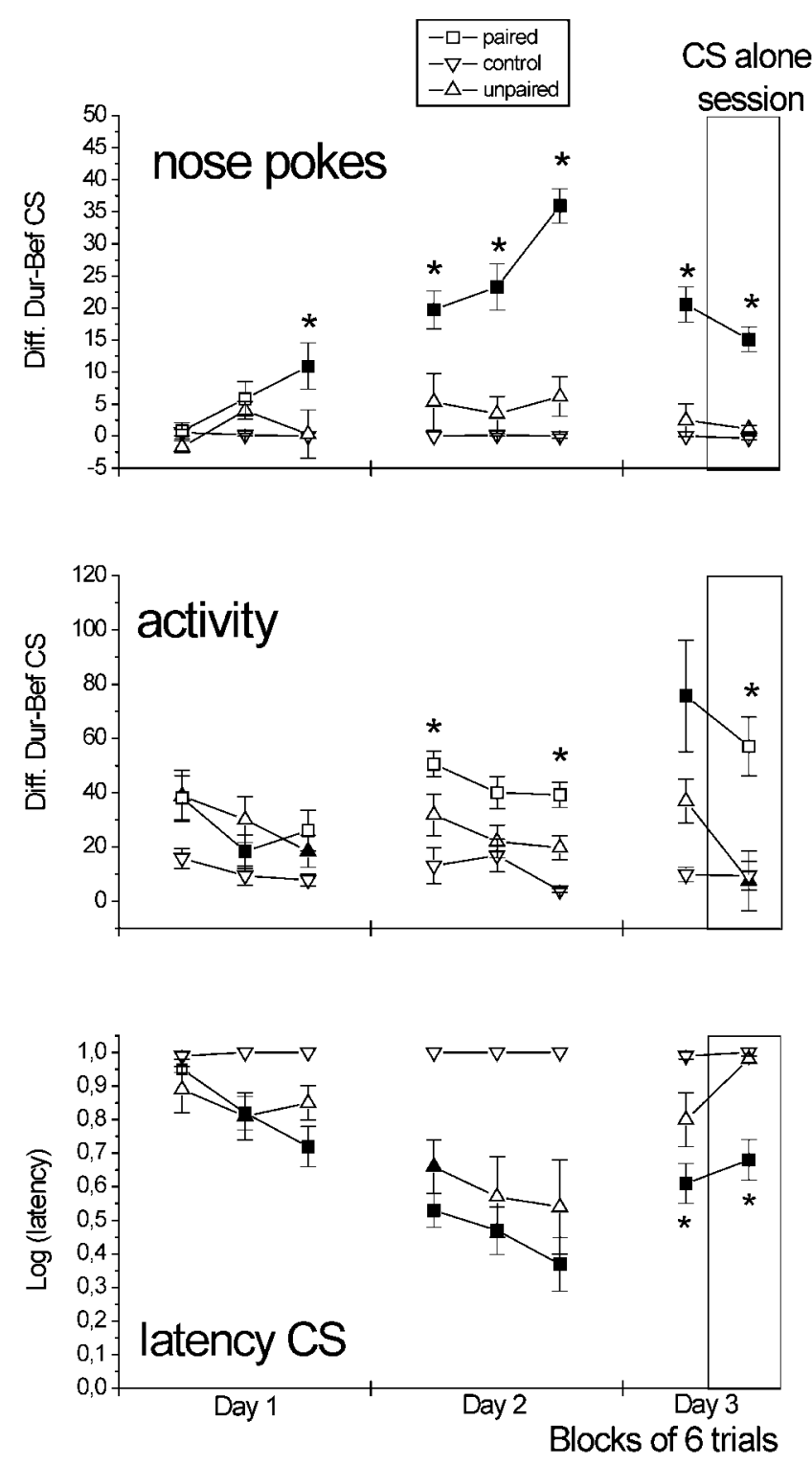

Figure 1. Behavioral effects of classical conditioning in experiment 1. Rats were fed ad libitum and received two pellets as the US. The paired $(n=11)$ and unpaired $(n=6)$ groups were given seven CS plus US sessions and one CS session. The control group $(n=10)$ received CS sessions only. Microdialysis measurements were performed on day 3 . Values of nose pokes and locomotor activity are expressed as the difference between the 10 sec during the $C S$ and the 10 sec before the CS. Latency is presented in the following logarithm form: value 1 equals the $10 \mathrm{sec}$ duration of the CS presentation. Error bars indicate means \pm SEM. Filled symbols indicate significant differences from the initial session. An asterisk indicates a significant difference from the unpaired group.

ANOVA with repeated measures was applied with sample as the withinsubject factor and group as the between-subject factor. When indicated, between-subject effects were analyzed using SNK tests. ANOVA with repeated measures for separate groups was followed, when indicated, by simple contrast analysis to compare the final basal sample with the subsequent samples.

\section{Results}

Behavior

Experiment 1

In the paired group, the frequency of nose pokes during the CS plus US presentations showed a steady increase, whereas the latency measure showed a decrease. This finding indicates that con- 
ditioning to the CS took place. Nose pokes were almost absent in the control group but showed a slight increase during CS presentation in the unpaired group. Latency values of the latter group showed a pattern that was similar to that seen for the paired group, without reaching their lowest values (Fig. 1).

A comparative statistical analysis showed main within-subject effects of session for all three measures; main between-subject effects of group for nose pokes $\left(F_{(2,24)}=43.548 ; p<0.001\right)$, activity $\left(F_{(2,23)}=9.502 ; p=0.001\right)$, and latency $\left(F_{(2,24)}=31.670\right.$; $p<0.001)$; and significant session by group interactions for nose pokes $\left(F_{(14,168)}=11.741 ; p<0.001\right)$, activity $\left(F_{(14,161)}=3.308\right.$; $p=0.006)$, and latency $\left(F_{(14,168)}=6.992 ; p<0.001\right)$. From the third session, the number of nose pokes in the paired group was different from those in the control and the unpaired groups.

Separate analysis of the three groups showed main withinsubject effects for nose pokes $\left(F_{(7,70)}=24.379 ; p<0.001\right)$, activity $\left(F_{(7,70)}=5.393 ; p=0.011\right)$, and latency $\left(F_{(7,70)}=16.767 ; p<\right.$ $0.001)$ for the paired group and within-subject effects for activity $\left(F_{(7,35)}=4.105 ; p=0.002\right)$ and latency $\left(F_{(7,35)}=4.289 ; p=\right.$ $0.024)$ for the unpaired group, but none for the control group. In the paired group, the number of nose pokes in the third and following sessions was increased compared with the first session, and the latency in the second and following sessions was decreased.

Analysis of the final (seventh) CS plus US session and the subsequent CS-alone session revealed a decrease in activity $\left(F_{(1,5)}\right.$ $=14.428 ; p=0.013)$ only in the unpaired group. This indicated that the responses to the CS in the unpaired animals were hardly associated with the subsequent US. The rats in the paired group continued to check the food dispenser for the expected pellets immediately at the start of the CS, although the frequency of nose pokes decreased slightly.

\section{Experiment 2}

The conditioned behavior of the rats in experiment 2 was similar to that in experiment 1 . Highly significant session by group interactions (nose pokes, $F_{(14,112)}=5.026$; activity, $F_{(14,112)}=$ 5.127; both, $p<0.001$ ), and between-subject group effects (nose pokes, $F_{(2,16)}=13.344$; activity, $F_{(2,16)}=13.535$; both, $\left.p<0.001\right)$ were obtained. Differences between groups were the same as those described for experiment 1 .

Separate analysis of the three groups showed main withinsubject effects for nose pokes $\left(F_{(7,35)}=7.413 ; p=0.002\right)$, activity $\left(F_{(7,35)}=7.757 ; p<0.001\right)$, and latency $\left(F_{(7,35)}=4.842 ; p=\right.$ $0.001)$ for the paired group and main within-subject effects for nose pokes $\left(F_{(7,49)}=2.584 ; p=0.024\right)$ and activity $\left(F_{(7,49)}=\right.$ $2.540 ; p=0.037$ ) for the unpaired group (the latency data were lost for the first $6 \mathrm{~d}$ in this group).

\section{Neurochemistry}

Basal NA and DA concentrations were $5.71 \pm 0.58$ and $1.74 \pm$ $0.29 \mathrm{pg} / 50 \mu \mathrm{l}$, respectively, in experiment 1 , and $5.25 \pm 0.47$ and $2.29 \pm 0.26 \mathrm{pg} / 50 \mu \mathrm{l}$, respectively, in experiment 2 . These values are based on the data obtained from rats with bilateral functioning microdialysis cannulas $(n=15$ in both experiment 1 and experiment 2 ). In the other rats (11 in experiment 1 and 5 in experiment 2), only one probe was functioning.

\section{Experiment 1}

Between-subject comparisons revealed significant sample by group interactions in the CS plus US session for both NA and DA $\left(F_{(6,72)}=5.526\right.$ and 6.266, respectively; both, $\left.p<0.001\right)$ and a group effect for DA $\left(F_{(2,24)}=7.443 ; p=0.003\right)$. In the fifth
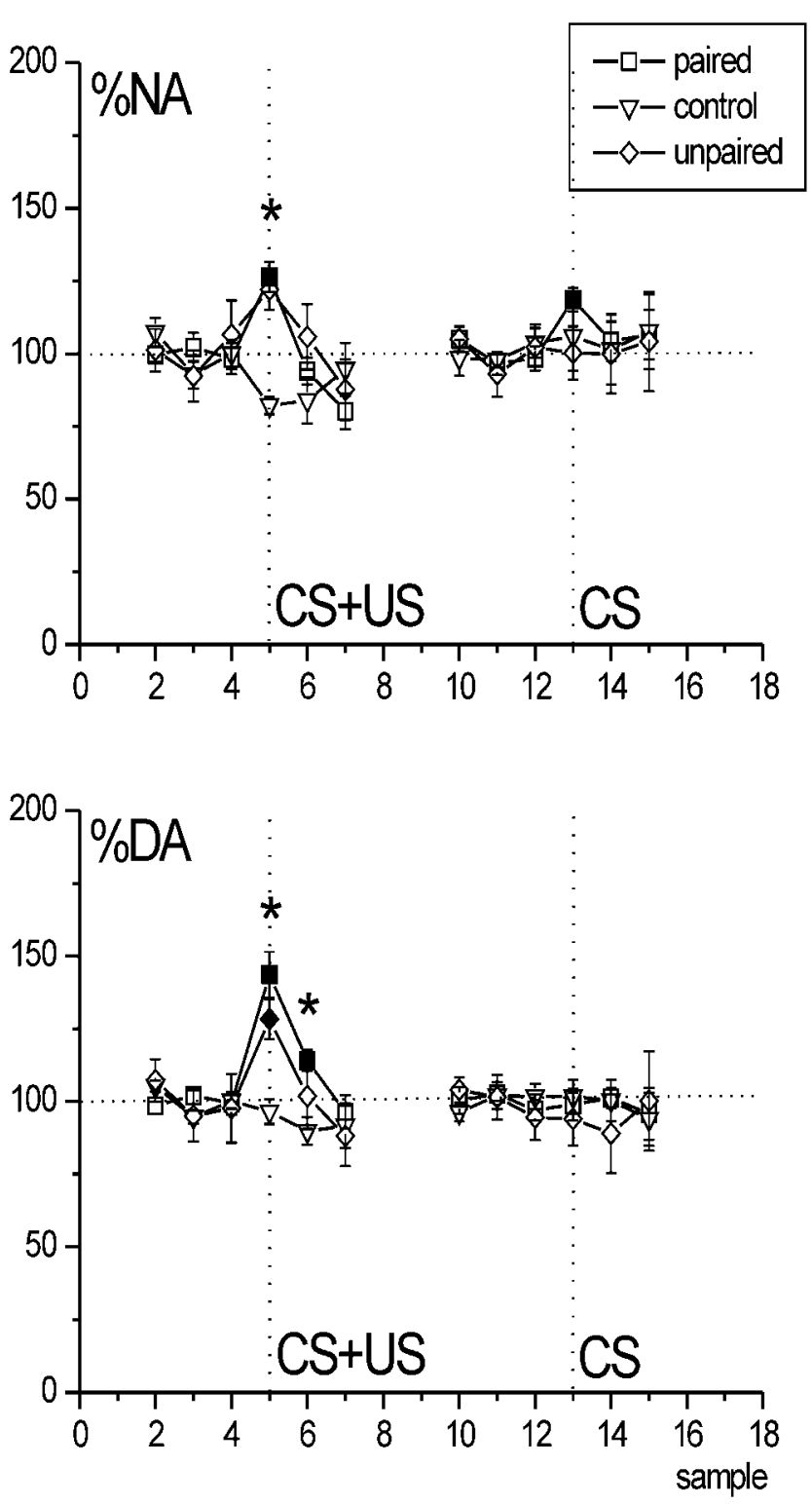

Figure 2. DA and NA efflux in the medial prefrontal cortex of rats fed ad libitum in experiment 1 and measured on day 3. The paired $(n=11)$ and unpaired $(n=6)$ groups were subjected to a CS plus US session and a CS session (extinction). The control group ( $n=10)$ received only $C S$ sessions. Error bars indicate means \pm SEM. Filled symbols indicate significant differences from preceding basal levels. An asterisk indicates a significant difference from the control group.

sample (taken during the session), NA and DA in the control group were significantly lower than in the other groups. Presentation of both the CS and the US increased NA and DA efflux in the paired group $\left(F_{(3,30)}=16.811\right.$ and 26.566 , respectively; $p<$ 0.001 for both) to a maximum of $126.4 \%$ (NA) and $144.0 \%$ (DA). In the unpaired group, NA $(122.1 \%)$ was not significantly altered, but DA was increased $\left(F_{(3,15)}=8.651 ; p=0.006\right)$ to $128.3 \%$. No effects of sample were observed in the controls (Fig. 2).

Measurements during the CS-alone session indicated no significant between-subject effects. Separate analysis revealed a small increase for NA in the paired group (to $118.6 \% ; F_{(1,10)}=$ 9.657; $p=0.011$ )

\section{Experiment 2}

Presentation of both the CS and the US resulted in significant sample by group interactions for both NA $\left(F_{(6,54)}=4.298 ; p=\right.$ 

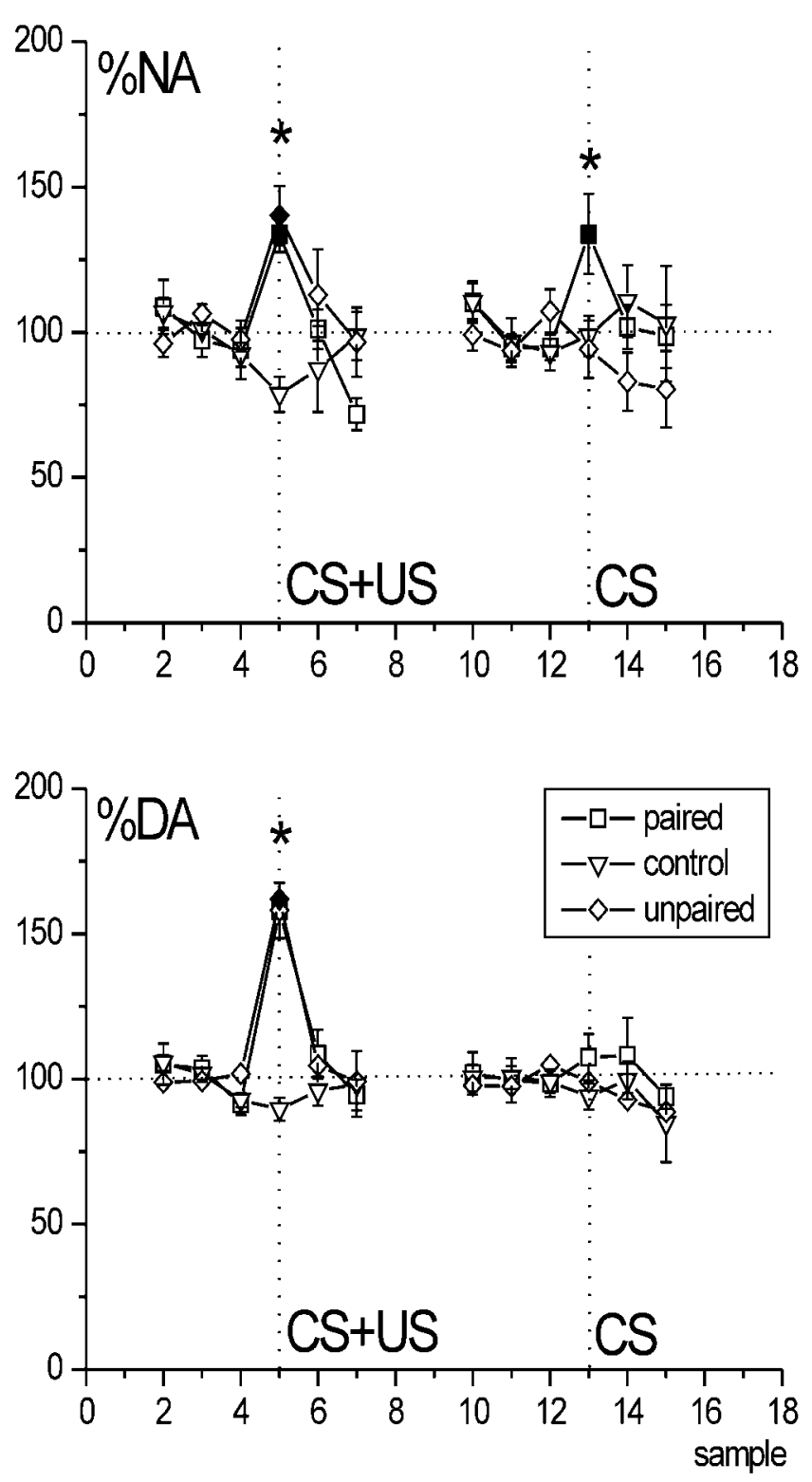

Figure 3. DA and NA efflux in the medial prefrontal cortex of food-restricted rats in experiment 2 that were measured on day 3 (paired group, $n=6$; unpaired group, $n=9$; control group, $n=6$ ). See Figure 2 for further explanation. The asterisk indicates a significant difference from the control group (CS plus US session) or from the control and unpaired groups (CS session). Error bars indicate means \pm SEM.

$0.003)$ and DA $\left(F_{(6,54)}=3.935 ; p=0.004\right)$. In the fifth sample (taken during the session), NA and DA were significantly lower in the control group compared with the other groups (Fig. 3).

Both NA and DA efflux were increased in the paired group $\left(F_{(3,15)}=16.848\right.$ and 29.123 , respectively; $\left.p<0.001\right)$ to a maximum of $133.7 \%$ (NA) and $158.0 \%$ (DA) and in the unpaired group to $140.2 \%$ (NA) and $161.9 \%$ (DA) $\left(F_{(3,24)}=5.370\right.$ and $29.123, p=0.015$ and 0.001 , respectively). No effects of sample were observed in the controls.

Measurements during the CS-alone session resulted in a significant sample by group interaction for $\mathrm{NA}\left(F_{(6,54)}=4.186 ; p=\right.$ $0.002)$. In the sample taken during the session, NA was significantly higher in the paired group compared with the other groups. Separate analyses indicated an increase only for NA in the paired group (to $133.8 \% ; F_{(3,30)}=4.304 ; p=0.022$ ).

\section{Discussion}

For the first time, prefrontal NA and DA responses in rewardrelated associative learning were directly compared. The results were surprising, because they revealed that presentation of an explicit, auditory stimulus that was associated previously with reward presentation only increased NA efflux in the PFC of the rats. Although DA is often regarded as the monoamine that is most strongly implicated in motivational processes (Robbins and Everitt, 1996; Kalivas and Nakamura, 1999), no conditioned increases in DA efflux were found.

\section{Responses to CS plus US presentations}

In this conditioning paradigm, the neutral auditory stimulus explicitly paired with pellet presentations was able to evoke a conditioned behavioral response. With training, the frequency of nose pokes during the CS increased markedly compared with the period immediately before; the latency until the first nose poke after CS presentation decreased, and general locomotor activity increased. This behavior was contingent on the association between the CS and the US, because neither the control nor the unpaired group showed similar levels of responses to the CS.

The combined presentation of CS plus US (noise plus pellets) to the paired groups significantly increased NA and DA efflux. Neurochemical responses were very similar in rats in the unpaired group that received the same number of pellets and auditory stimuli as the rats in the paired group, but were completely absent in the control rats, which only received the auditory stimulus. This is consistent with our previous results with aversive stimuli (Feenstra et al., 2001). The measurements were performed during the seventh CS plus US session (trials 37-42), and the clear responses suggest that NA and DA responses did not habituate. This is in line with previous findings for DA (Bassareo and Di Chiara, 1997). Although habituation of responses of NA neurons was observed by Sara and Segal (1991), the fact that this occurred only after $>40$ trials within one session makes comparison with the present data difficult.

\section{Responses to CS-alone presentations}

Rats in the paired group reacted strongly to the presentation of the conditioned auditory stimulus. The extinction of the conditioned responses over the six unrewarded trials was not so rapid that the measures taken for the session were significantly decreased compared with the final CS plus US session. One day, extra training and an increased intertrial interval resulted in an apparently stronger conditioning but more rapid extinction (Cheng et al., 2003). The unpaired group lost any behavioral reaction to the CS, suggesting that in this respect, the procedure of explicitly not pairing the stimuli results in a lower strength of conditioning than the procedure of fully random presentation used by Cheng et al. (2003).

\section{Prefrontal cortex DA and appetitive conditioning}

Our experiments revealed an absence of a conditioned DA response even when the US magnitude and motivation (food restriction) were increased. This was unexpected, because DA conditioning was reported previously (Bassareo and Di Chiara, 1997; Ahn and Phillips, 1999). At first glance, this may indicate a lack of salience of our US, because both of the other groups presented special palatable snacks as the US, but we observed that rats, even when fed ad libitum, will eat large quantities of the reward pellets we used (M. G. Feenstra and M. H. Botterblom, unpublished observations). A possibly more important difference is that 
found in the CS salience. It is well known that the more salient or noticeable the CS, the more effective the conditioning (Schwartz, 1984). This depends on the intensity of the CS, on the CS-US similarity (an implicit cue is more effective than an explicit one), and on the spatial location of the CS (stimuli that appear in the same location as the US are associated more quickly). This mechanism may also apply to neurochemical responses, because several microdialysis studies showed a prefrontal DA activation when the CS consisted of the smell or sight of food (BertolucciD'Angio et al., 1990; Ahn and Phillips, 1999) or was presented in the same spatial location (Bassareo and Di Chiara, 1997). Compared with our results, it appears that more neutral and explicit cues, which have no spatial or physical similarity to food, do not readily induce such a conditional activation of prefrontal DA. A similar distinction was made by Saulskaya and Marsden (1995), who observed a higher responsiveness of accumbens DA to contextual over explicit cues associated previously with foot shocks. In apparent contrast to the lack of conditioned DA efflux in the medial PFC, we observed small but significant increases in DA efflux in nucleus accumbens subareas after presentation of an explicit auditory CS in food-restricted rats (Cheng et al., 2003). However, this can be explained by the finding that food restriction is known to alter both basal efflux and conditioned activation of accumbens DA, whereas no such findings were reported for the PFC (Pothos et al., 1995; Wilson et al., 1995).

\section{Prefrontal cortex NA and appetitive conditioning}

During the CS-alone session, extracellular concentrations of NA were increased in the PFC of rats. Although an increase was observed in both experiments, only in the group that was food restricted and trained with a higher-magnitude US was the obtained value different from that of the other groups. This may relate to the fact that increasing the conspicuousness of stimuli leads to incremental changes in NA efflux in the PFC (Dalley and Stanford, 1995) and neuronal activity in the locus ceruleus (LC) (Rasmussen et al., 1986; Grant et al., 1988). The only two publications that we found in the literature that relate to our study may be taken to support our findings, because they suggest the presence of a conditioned NA LC activation in food-deprived rats (Sara and Segal, 1991) but not in cats fed ad libitum (Rasmussen and Jacobs, 1986).

The LC NA system is thought to be an important determinant of attentional functions (for review, see Aston-Jones et al., 1999) and has been suggested to maintain selective attention onto explicit CSs (Selden et al., 1990; McQuade and Stanford, 2000). Exposure to such a cue leads to retrieval of the previously acquired information, to maintenance of an active representation of that information [working memory (Arnsten, 1997)], and, after reinforcement, to reconsolidation of the association (Sara, 2000). Given that activation of the locus ceruleus has been found to improve retrieval (Sara and Devauges, 1988), NA systems must contribute to the retrieval process.

\section{Concluding remarks}

The present study shows the presence of a differential activation of NA and DA efflux in the PFC after presentation of an auditory conditioned stimulus. CS presentation elicited a conditioned efflux of NA but not of DA. We propose that the (acquired) salience of the explicit stimulus was not sufficient to trigger an activation of the PFC DA system. When reward-predicting stimuli are processed, NA may play a role that is complementary to that of DA. The NA neurons may be more easily activated by the immediate attention-grabbing properties of explicit stimuli, whereas DA neurons may be more involved in coding the salience of stimuli in their context.

\section{References}

Ahn S, Phillips AG (1999) Dopaminergic correlates of sensory-specific satiety in the medial prefrontal cortex and nucleus accumbens of the rat. J Neurosci 19:RC29(1-6).

Arnsten AF (1997) Catecholamine regulation of the prefrontal cortex. J Psychopharmacol 11:151-162.

Aston-Jones G, Rajkowski J, Cohen J (1999) Role of locus coeruleus in attention and behavioral flexibility. Biol Psychiatry 46:1309-1320.

Bassareo V, Di Chiara G (1997) Differential influence of associative and nonassociative learning mechanisms on the responsiveness of prefrontal and accumbal dopamine transmission to food stimuli in rats fed ad libitum. J Neurosci 17:851-861.

Bertolucci-D’Angio M, Serrano A, Driscoll P, Scatton B (1990) Involvement of mesocorticolimbic dopaminergic systems in emotional states. Prog Brain Res 85:405-417.

Cheng JJ, de Bruin JP, Feenstra MG (2003) Dopamine efflux in nucleus accumbens shell and core in response to appetitive classical conditioning. Eur J Neurosci 18:1306-1314.

Dalley JW, Stanford SC (1995) Incremental changes in extracellular noradrenaline availability in the frontal cortex induced by naturalistic environmental stimuli: a microdialysis study in the freely moving rat. J Neurochem 65:2644-2651.

Drouin C, Darracq L, Trovero F, Blanc G, Glowinski J, Cotecchia S, Tassin J-P (2002) $\alpha 1$ b-Adrenergic receptors control locomotor and rewarding effects of psychostimulants and opiates. J Neurosci 22:2873-2884.

Feenstra MG (2000) Dopamine and noradrenaline release in the prefrontal cortex in relation to conditioned and unconditioned stress and reward. Prog Brain Res 126:133-163.

Feenstra MG, Botterblom MH, van Uum JF (1998) Local activation of metabotropic glutamate receptors inhibits the handling-induced increased release of dopamine in the nucleus accumbens, but not that of dopamine or noradrenaline in the prefrontal cortex: comparison with inhibition of ionotropic receptors. J Neurochem 70:1104-1113.

Feenstra MG, Vogel M, Botterblom MH, Joosten RN, de Bruin JP (2001) Dopamine and noradrenaline efflux in the rat prefrontal cortex after classical aversive conditioning to an auditory cue. Eur J Neurosci 13:1051-1054.

Feenstra MG, Botterblom MH, van Uum JF (2002) Behavioral arousal and increased dopamine efflux after blockade of NMDA-receptors in the prefrontal cortex are dependent on activation of glutamatergic neurotransmission. Neuropharmacology 42:752-763.

Goldman-Rakic PS, Muly III EC, Williams GV (2000) D(1) receptors in prefrontal cortex cells and circuits. Brain Res Brain Res Rev 31:295-301.

Grant ST, Aston-Jones G, Redmond DE (1988) Responses of primate locus coeruleus neurons to simple and complex sensory stimuli. Brain Res Bull 21:401-410.

Kalivas PW, Nakamura M (1999) Neural systems for behavioral activation and reward. Curr Opin Neurobiol 9:223-227.

McQuade R, Stanford SC (2000) A microdialysis study of the noradrenergic response in rat frontal cortex and hypothalamus to a conditioned cue for aversive, naturalistic environmental stimuli. Psychopharmacology (Berl) 148:201-208.

Norušis MJ (1990) SPSS advanced statistics user's guide, pp 255-293. Chicago: SPSS.

Pothos EN, Creese I, Hoebel BG (1995) Restricted eating with weight loss selectively decreases extracellular dopamine in the nucleus accumbens and alters dopamine response to amphetamine, morphine, and food intake. J Neurosci 15:6640-6650.

Rasmussen K, Jacobs BL (1986) Single unit activity of locus coeruleus neurons in the freely moving cat. II. Conditioning and pharmacologic studies. Brain Res 371:335-344.

Rasmussen K, Morilak DA, Jacobs BL (1986) Single unit activity of locus coeruleus neurons in the freely moving cat. I. During naturalistic behaviors and in response to simple and complex stimuli. Brain Res 371:324-334. 
Robbins TW, Everitt B (1996) Neurobehavioural mechanisms of reward and motivation. Curr Opin Neurobiol 6:228-236.

Sara SJ (2000) Strengthening the shaky trace through retrieval. Nat Rev Neurosci 1:212-213.

Sara SJ, Devauges V (1988) Priming stimulation of the locus coeruleus facilitates memory retrieval in the rat. Brain Res 438:299-303.

Sara SJ, Segal M (1991) Plasticity of sensory responses of locus coeruleus neurons in the behaving rat: implications for cognition. Prog Brain Res $88: 571-585$.

Saulskaya N, Marsden CA (1995) Conditioned dopamine release: dependence upon $N$-methyl-D-aspartate receptors. Neuroscience 67:57-63.

Schwartz B (1984) Psychology of learning and behavior, pp 126-138. New York: Norton.
Selden NR, Robbins TW, Everitt BJ (1990) Enhanced behavioral conditioning to context and impaired behavioral and neuroendocrine responses to conditioned stimuli following ceruleocortical noradrenergic lesions: support for an attentional hypothesis of central noradrenergic function. J Neurosci 10:531-539.

Ventura R, Cabib S, Alcaro A, Orsini C, Puglisis-Allegra S (2003) Norepinephrine in the prefrontal cortex is critical for amphetamineinduced reward and mesoaccumbens dopamine release. J Neurosci 23:1879-1885

Wilson C, Nomikos GG, Collu M, Fibiger H (1995) Dopaminergic correlates of motivated behavior: importance of a drive. J Neurosci 15: $5169-5178$. 